\title{
A new biomechanical classification system for split fractures of the humeral greater tuberosity: guidelines for surgical treatment
}

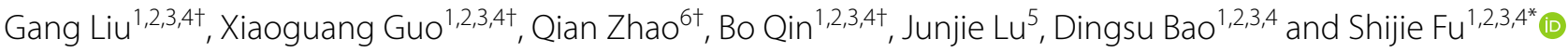

\begin{abstract}
Background: Split fractures of the humeral greater tuberosity (HGT) are common injuries. Although there are numerous surgical treatments for these fractures, no classification system combining clinical and biomechanical characteristics has been presented to guide the choice of fixation method.

Methods: We created a standardised fracture of the HGT in 24 formalin-fixed cadavers. Six were left as single-fragment fractures (Group A), six were further prepared to create single-fragment with medium size full-thickness rotator cuff tear (FT-RCT) fractures (Group B), six were cut to create multi-fragment fractures (Group C), and six were cut to create multi-fragment with FT-RCT fractures (Group D). Each specimen was fixed with a shortened proximal humeral internal locking system (PHILOS) plate. The fixed fractures were subjected to load and load-to-failure tests and the differences between groups analysed.
\end{abstract}

Results: The mean load-to-failure values were significantly different between groups (Group A, $446.83 \pm 38.98 \mathrm{~N}$; Group B, 384.17 \pm 36.15 N; Group C, 317.17 $\pm 23.32 \mathrm{~N}$ and Group D, $266.83 \pm 37.65 \mathrm{~N}, P<0.05)$. The load-to-failure values for fractures with a greater tuberosity displacement of $10 \mathrm{~mm}$ were significantly different between each group (Group A, 194.00 \pm 29.23 N; Group B, $157.00 \pm 29.97$ N; Group C, $109.00 \pm 17.64$ N and Group D, 79.67.83 \pm 15.50 N; $P<0.05$ ). These findings indicate that fractures with a displacement of $10 \mathrm{~mm}$ have different characteristics and should be considered separately from other HGT fractures when deciding surgical treatment.

Conclusions: Biomechanical classification of split fractures of the HGT is a reliable method of categorising these fractures in order to decide surgical treatment. Our findings and proposed system will be a useful to guide the choice of surgical technique for the treatment of fractures of the HGT.

Keywords: Humeral greater tuberosity, Split fracture, Biomechanical classification, Rotator cuff tear, Surgical technique

*Correspondence: fushijieggj@126.com

† Gang Liu, Xiaoguang Guo, Qian Zhao and Bo Qin contributed equally to this work

${ }^{1}$ Department of Orthopedics, Affiliated Traditional Chinese Medicine Hospital of Southwest Medical University, Luzhou, Sichuan, China

Full list of author information is available at the end of the article

\section{Background}

The humeral greater tuberosity (HGT) is the attachment point of the rotator cuff, which is the axis of the shoulder and plays an important role in shoulder movement. Proximal humeral fractures (PHFs) are the third most common fractures in elderly individuals, accounting for $5 \%$ of all fractures among such patients $[1,2]$. In contrast, fractures of the HGT occur more frequently in younger patients with strong bones following original author(s) and the source, provide a link to the Creative Commons licence, and indicate if changes were made. The images or other third party material in this article are included in the article's Creative Commons licence, unless indicated otherwise in a credit line to the material. If material is not included in the article's Creative Commons licence and your intended use is not permitted by statutory regulation or exceeds the permitted use, you will need to obtain permission directly from the copyright holder. To view a copy of this licence, visit http://creativecommons.org/licenses/by/4.0/. The Creative Commons Public Domain Dedication waiver (http://creativeco mmons.org/publicdomain/zero/1.0/) applies to the data made available in this article, unless otherwise stated in a credit line to the data. 
high-velocity trauma. These fractures are common and account for up to $20 \%$ of all PHFs [3, 4].

There are three classification systems that are routinely used to assess GT fractures; namely, the Neer, AO and morphological classification systems [5-7]. The Neer system classifies PHFs into four categories based on location: the GT, the lesser tuberosity, the humeral head or the humeral shaft if there is displacement of $>1 \mathrm{~cm}$ or angulation of $>45$. However, in 2005, Kim reported that isolated GT fractures have different characteristics to other PHFs; thus, the treatment and classification of these fractures should be different to that of other PHFs [8]. The AO system classifies GT fractures as non-displaced, displaced or associated with shoulder dislocation. Recent studies on split fracture have focused mostly on morphological classification. In 2014, Mutch proposed a classification system dividing GT fractures into three types: avulsion, split or depressed. Split fractures of the GT (Fig. 1) are the most common, accounting for $41 \%$ of all GT fractures. Yet, there is currently no classification system for split fractures of the HGT with rotator cuff tear (RCT) which considers the number of fragments.

Coexisting soft tissue lesions of greater tuberosity fractures were discussed in several reports as being possible indications for surgical treatment and that might result in persistent late pain, shoulder dysfunction $[9,10]$. Early in 1996, Gary reported a non-union of the greater tuberosity fracture concomitant with full-thickness rotator cuff tear and it was successfully treated with arthroscopy [11]. Gumina described 24 patients with missed greater tuberosity fractures, of which 11 (45.8\%) had rotator cuff tears with the use of magnetic resonance imaging (MRI) for detecting soft tissue pathologies [12]. Moreover, Eran Maman reported rotator cuff tears were the most commonly diagnosed pathology of coexisting lesions with GT fractures, of which the supraspinatus was the most frequently involved tendon (36\% of all pathologies). He believes that it is important to identify and repair pathologies concomitant with GT fractures [13].

Despite their high prevalence, coexisting Lesions have largely been ignored. There have been few clinical guidelines on comminuted fractures or RCT in the context of fractures of the HGT. According to our clinical data findings from January 2010 to January 2018 (Fig. 2), such injuries have different shoulder function outcomes; particularly fragmented fractures or those combined with RCT. The present study sought to address the following questions: are there any significant differences between fractures that are categorised according to specific clinical features? What are the optimal treatments when these differences are considered? We created four cadaver models (Fig. 3),

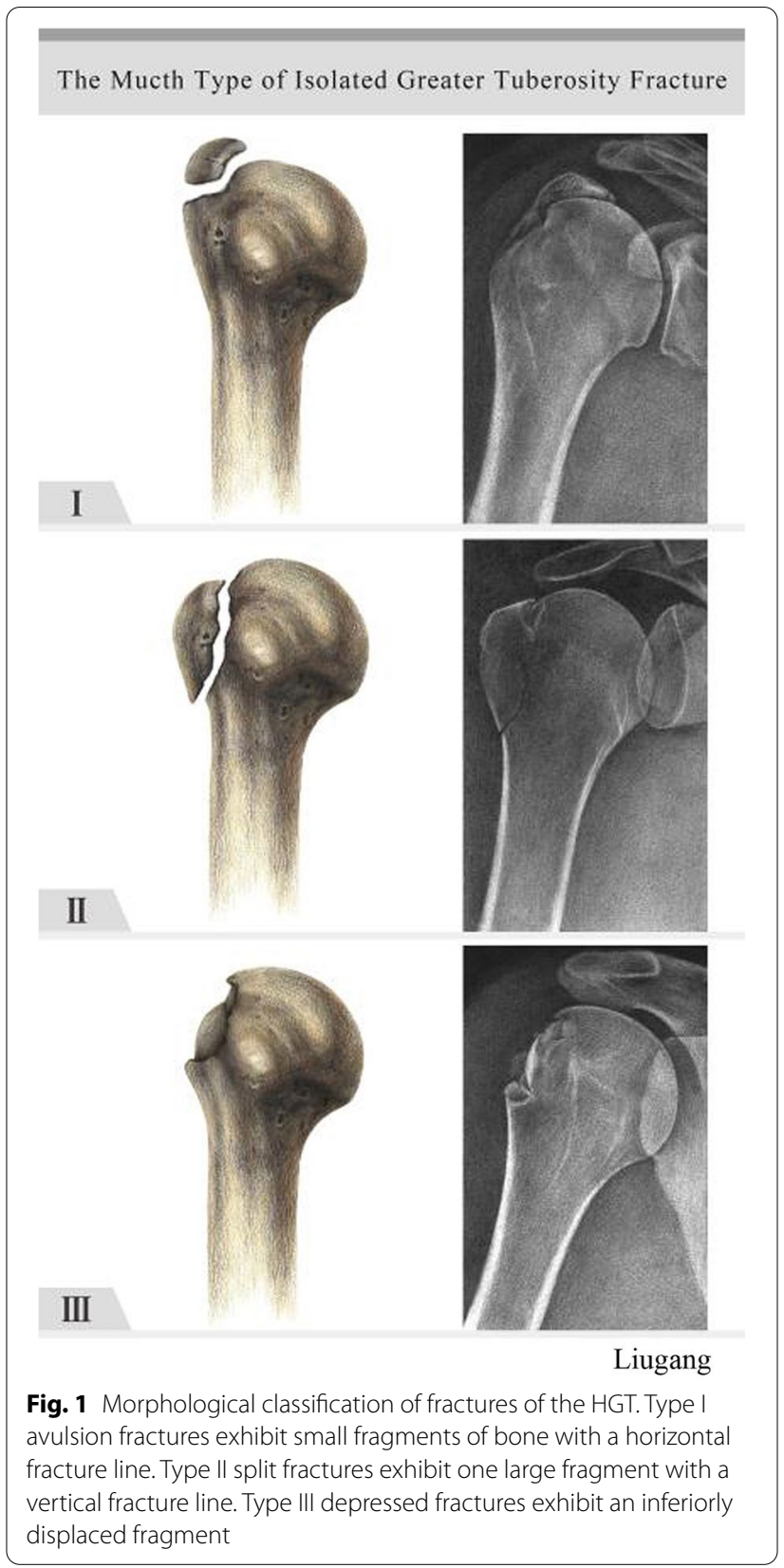

evaluated whether there are significant differences between the models and identified optimal operative treatments for each.

The aims of this study were to: (i) create four cadaver models from our long-term clinical data (Fig. 3); (ii) identify the different characteristics of each model and (iii) identify the optimal treatment for different types of fractures. The primary hypothesis was that biomechanical classification of fractures of the HGT is a reliable system and can be used to guide the choice of surgical technique. 

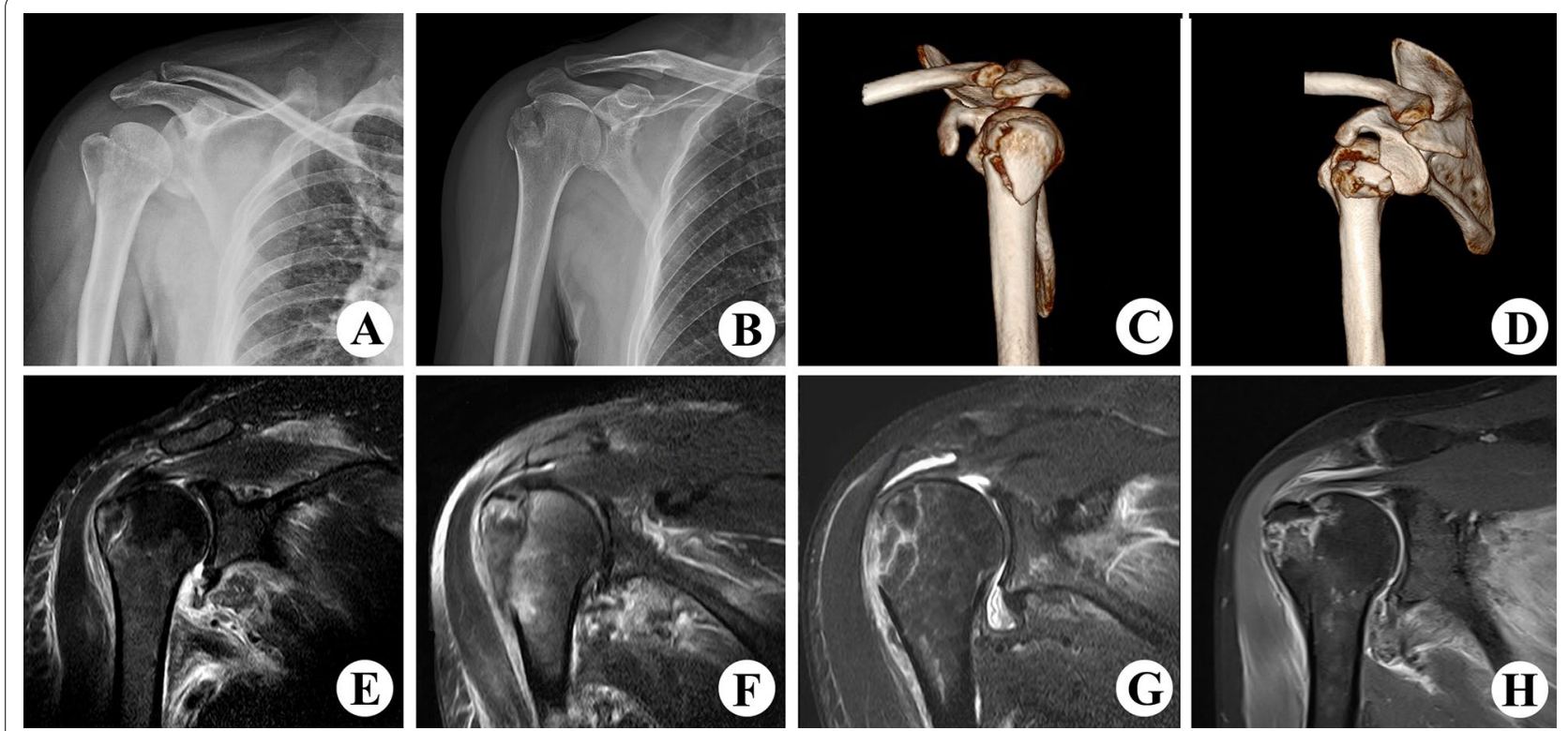

Fig. 2 Clinical imaging findings of fractures of the humeral greater tuberosity. A X-ray image of a single-fragment fracture. B X-ray image of a multi-fragment fracture. C Computed tomography scan of a single-fragment fracture. D Computed tomography scan of a multi-fragment fracture. E Magnetic resonance image of a single-fragment fracture. F Magnetic resonance image of a single-fragment fracture with rotator cuff tear. $\mathbf{G}$ Magnetic resonance image of a multi-fragment fracture without rotator cuff tear. $\mathbf{H}$ Magnetic resonance image of a multi-fragment fracture with rotator cuff tear

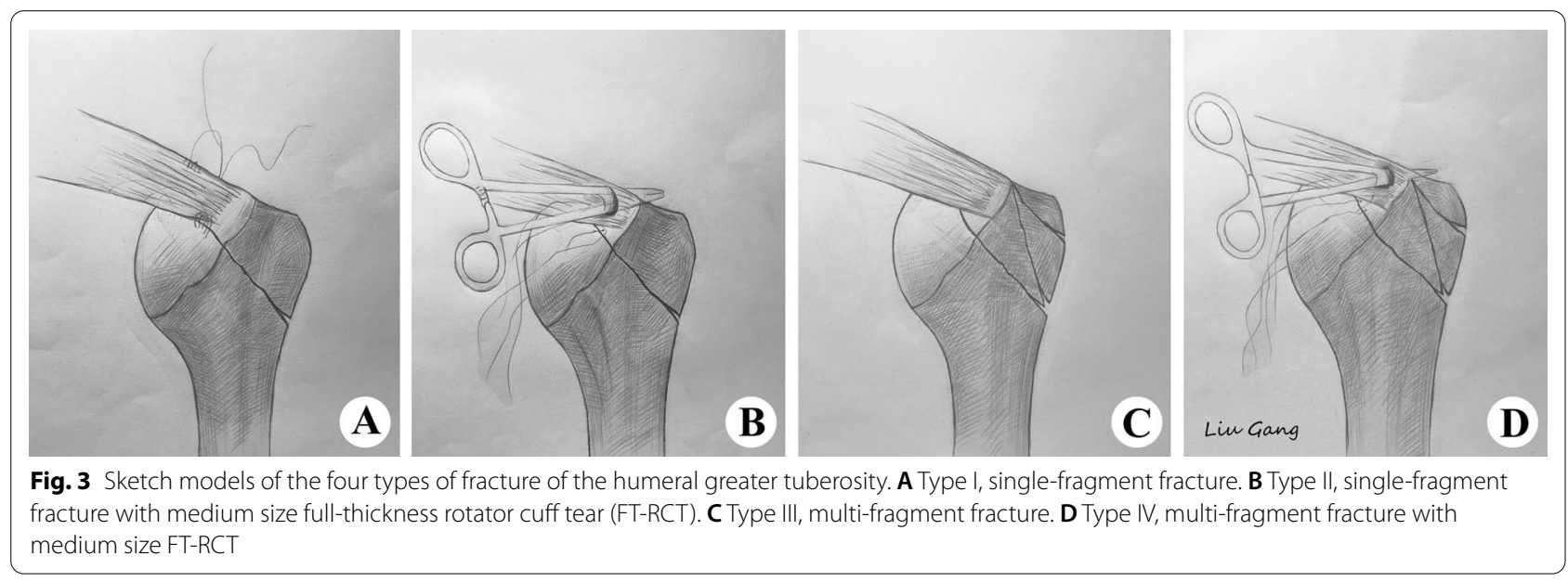

\section{Methods}

\section{Specimen selection and preparation}

This study was conducted in the Biomechanical and Anatomy Laboratory of the South-west Medical University, Sichuan, China. We selected donors whose family had given written consent for the donation of their to science. Inclusion criteria were cadavers from Chinese patients that had: (i) been soaking in the same formalin mixture for 6 months, (ii) full-grown and normal shoulder joints, (iii) no history of previous shoulder operations, (iv) no history and/or signs of previous fracture, (v) a cadaveric age of less than 60 years and (vi) normal BMD. Exclusion criteria were: (i) history of diabetes or smoking, (ii) history of soft tissue injury in the AC or shoulder joint (e.g. osteoarthritis, shoulder instability or $\mathrm{RCT}$ ) and (iii) incomplete specimens.

Bone density was assessed by X-ray (OSTEOCORE-3; Golden, China), and BMD was compared between the four groups using one-way analysis of variance (ANOVA) to ensure that there were no significant differences in 
BMD, which could affect the biomechanical results. Finally, 24 formalin-fixed (35\% formaldehyde in alcohol; Da-pin chemical industry, Guangzhou, China) cadaver shoulder specimens (16 right and 8 left; 16 men and 8 women) were used for this study.

To prepare the specimens, we resected all soft tissue from the scapula and humerus, retaining only the whole humerus and rotator cuff tendon (Fig. 4A). Parts of the supraspinatus and tendon of $>5 \mathrm{~cm}$ in length were preserved at the bony insertion. We created a standardised Type I GT fracture at an angle of $50^{\circ}$ to the shaft of the humerus using a thin-blade reciprocating saw (Guoke, China) as previously described [14] (Fig. 4B). Six models were left as Type I fractures (Group A), while 12 were further cut on both sides of the single-fragment to create multi-fragment fractures (Fig. 4C). Finally, we chose six single- and six multi-fragment models to be used to create models with medium size (i.e. $1-3 \mathrm{~cm}$ according to the Cofield Classification), full-thickness (FT) RCT [15]. The thickness and width of the supraspinatus were measured using digital callipers, and the supraspinatus footprint area was marked and measured.

\section{Fixation configurations}

We chose to modify a small locking plate (a shortened PHILOS plate, HS-A-BU0173-029, China; Fig. 4D) to fix the fractures, in line with guidelines for the surgical treatment of displaced fractures of the HGT [16]. All operations were performed by the senior author (Shijie-Fu). The shortened PHILOS plate was prepared by cutting off the three-hole screw on the plate shaft using strong scissors (Guoke, China). Then, fragments were accurately fixed using K-wires. The PHILOS plate was fixed at $5 \mathrm{~mm}$ below the top of the GT and the medial intertubercular sulcus, according to the protocol of Ali-jabran [17]. We fixed the medium size $(1.5-\mathrm{cm})$ FT-RCT of Groups B and D by suturing both sides of the supraspinatus (Fig. 4F) using Ethicon 5\#, a high-polymer polyethylene (Johnson, USA).

\section{Load test}

Specimens were placed in a special clamp (Fig. 5) to ensure stability during the load test. All tests were performed at room temperature, and the surface of the prepared-modal was kept constantly moist with
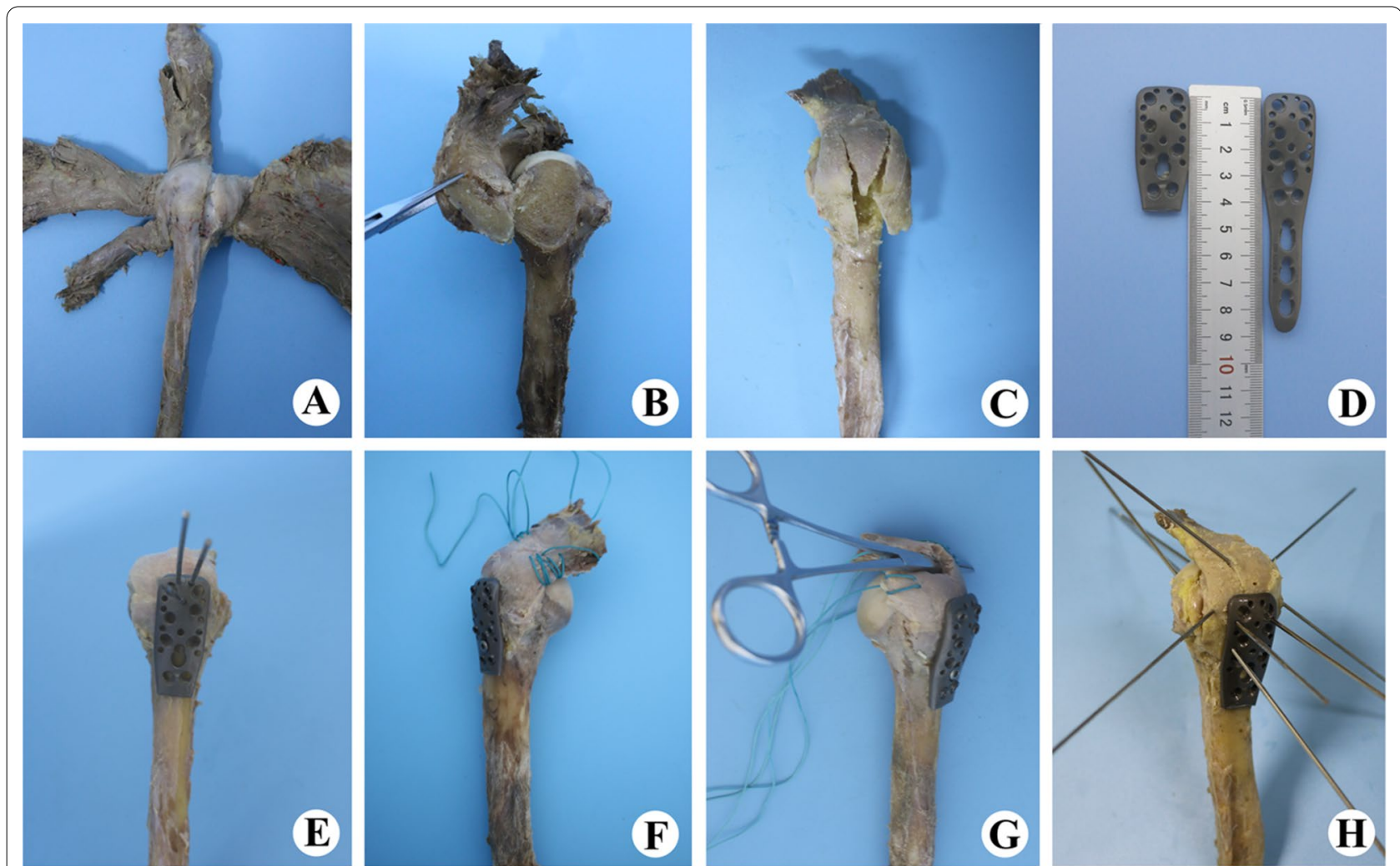

Fig. 4 Diagram showing the preparation of specimen models. A The whole humerus and rotator cuff tendon were retained. B A standardised greater tuberosity fracture. C The single-fragment fracture was cut into multiple fragments. D Comparison of the PHILOS and shortened PHILOS plates. E Standardised fixation of single-fragment fractures. $\mathbf{F}$ Both sides of the supraspinatus were sutured using Ethicon $5 \#$ Johnson suture material. G A medium size, full-thickness rotator cuff tear was created. $\mathbf{H}$ Fixation of multi-fragment fractures 

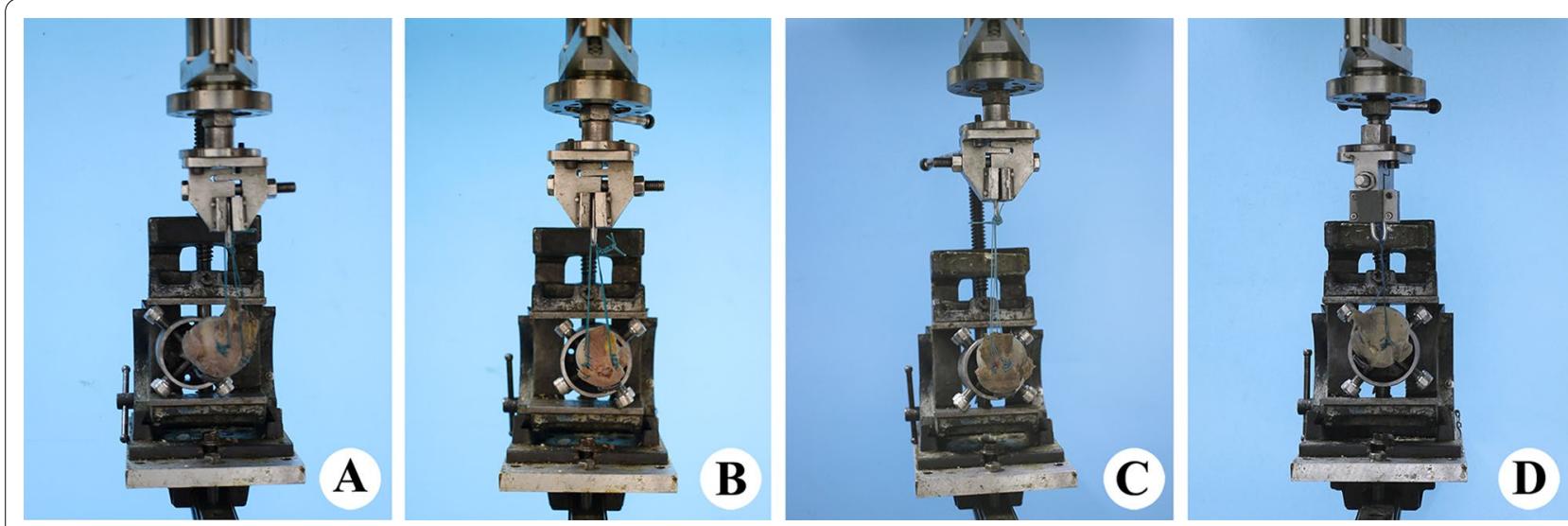

Fig. 5 Diagrams of the load and load-to-failure tests. A Group A, single-fragment fracture. B Group B, single-fragment fracture with medium size full-thickness rotator cuff tear (FT-RCT). C Group C, multi-fragment fracture. D Group D, multi-fragment fracture with medium size FT-RCT

isotonic saline. One side of the specimen was fixed to the biomechanical testing machine (Bose Electro Force 3520-AT, USA), and the other was fixed to the upper part of the testing machine (Fig. 5). During mechanical tests, the supraspinatus was fixed at an angle of $90^{\circ}$ abduction. A superior preload of $50 \mathrm{~N}$ was then applied out to assess the time effect, stress relaxation and stability of specimen fixation. The distance at $5 \mathrm{~N}$ was set as the initial reference (starting point). The electrodynamic testing machine applied a load at a constant speed of $5 \mathrm{~N} / \mathrm{s}$. The load test was repeated 10 times with intervals of 3 min to avoid stress fatigue.

\section{Load-to-failure test}

Tests to assess ultimate failure load $(\mathrm{N})$ were performed at a constant speed of $1 \mathrm{~mm} / \mathrm{min}$ in the superior-inferior direction, and the mode of failure was recorded. We also recorded results for fractures with 3, 5 and $10-\mathrm{mm}$ displacement of the HGT. Failure was defined as RCT rupture, internal fixation failure or complete dislocation of HGT.

\section{Statistical analysis}

The Statistical Package for the Social Sciences (SPSS) 19.0 software (Chicago, IL, USA) was used for all statistical analyses. All data are presented as mean \pm standard deviation $(\bar{x} \pm s)$. Homogeneity of variance was evaluated using the Shapiro-Wilk test. One-way ANOVA was used for multiple comparisons between groups when the variances were homogeneous. A significance level of $P<0.05$ was accepted as statistically significant.

\section{Results}

\section{Specimens and basic physical properties}

The mean age of the cadavers at the time of death was 43.5 (range: $29-52$ ) years. There were no significant differences in BMD, supraspinatus thickness, tendon width, footprint thickness or footprint width between the groups (Table 1).

\section{Displacement of fractures of the humeral greater tuberosity}

Results relating to 3-, 5- and 10-mm displacement of fractures of the HGT are summarised in Table 2. A steady but significant decrease was observed among the fractures with $10 \mathrm{~mm}$ displacement from Groups A to D. However,

Table 1 Basic physical properties of the cadaver specimens were as follows

\begin{tabular}{lrrrr}
\hline Physical properties & \multicolumn{1}{c}{ Group A } & Group B & Group C & Group D \\
\hline BMD, g/cm & $0.52 \pm 0.03$ & $0.52 \pm 0.05$ & $0.51 \pm 0.06$ & $0.52 \pm 0.05$ \\
SS thickness (mm) & $5.25 \pm 0.42$ & $5.37 \pm 0.57$ & $5.01 \pm 0.67$ & $5.34 \pm 0.37$ \\
SS width (mm) & $24.23 \pm 2.53$ & $24.15 \pm 2.52$ & $23.73 \pm 2.37$ & $24.08 \pm 2.26$ \\
Footprint length (mm) & $12.36 \pm 1.88$ & $12.26 \pm 1.41$ & $12.42 \pm 1.19$ & $11.96 \pm 1.51$ \\
Footprint width (mm) & $23.01 \pm 1.52$ & $22.59 \pm 1.78$ & $22.35 \pm 1.49$ & $23.43 \pm 2.01$ \\
\hline
\end{tabular}

Group $A(n)=B=C=D=6, B M D$ bone mineral density, SS supraspinatus 
Table 2 The findings of $3 \mathrm{~mm}, 5 \mathrm{~mm}$ and $10 \mathrm{~mm}$ HGT displacements

\begin{tabular}{lcccc}
\hline Displacement $(\mathbf{m m})$ & Group A (N) & Group B (N) & Group C (N) & Group D (N) \\
\hline 3 & $33.50 \pm 3.39^{\mathrm{bc}}$ & $31.17 \pm 6.24$ & $27.17 \pm 5.34$ & $27.00 \pm 3.74$ \\
5 & $80.17 \pm 10.01^{\mathrm{bc}}$ & $66.83 \pm 17.80^{\mathrm{bc}}$ & $45.00 \pm 5.83$ & $41.50 \pm 8.19$ \\
10 & $194.00 \pm 29.23^{\mathrm{abc}}$ & $157.00 \pm 29.97^{\mathrm{bc}}$ & $109.00 \pm 17.64^{\mathrm{c}}$ & $79.67 \pm 15.50$ \\
\hline
\end{tabular}

Each group contained six specimens, a: versus Group B, b: versus Group C, c: versus Group D $(P<0.05)$

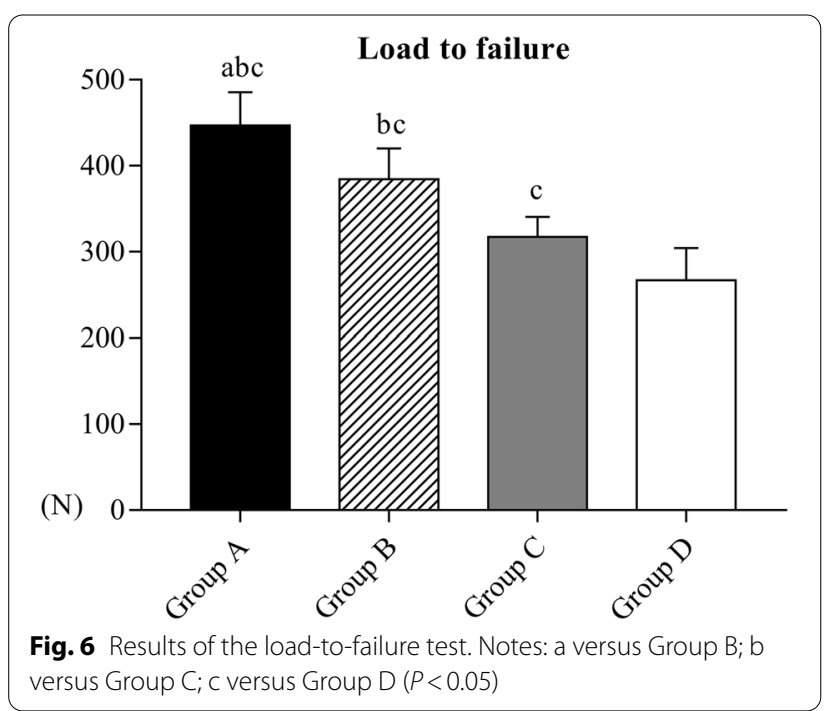

there were no statistically significant differences between the fractures with $10 \mathrm{~mm}$ displacement and those with 3or 5-mm displacement within any group.

\section{Load-to-failure}

Of the four groups, Group A had the highest mean loadto-failure value $(446.83 \pm 38.98 \mathrm{~N})$, and the mean value was significantly different between each group (Group B, Type II fracture, $384.17 \pm 36.15 \mathrm{~N}$; Group C, Type III fracture, $317.17 \pm 23.32 \mathrm{~N}$ and Group D, Type IV fracture, $266.83 \pm 37.65 \mathrm{~N}$ ) (Fig. 6). The mode of failure was fracture at the humeral surgical neck in six cases of Group A. Failure was humeral surgical neck fracture in five cases and GT fragment displacement in one case of Group B. In Group C, the mode of failure was GT fragment pulled out in two cases, surgical neck fracture in three cases and anatomic neck fracture in one. In Group $\mathrm{D}$, one case failed due to rotator cuff rupture, two due to GT- fragment pulled out and three due to surgical neck fracture.

\section{Discussion}

Our study demonstrates that there are significant differences between the characteristics of HGT fractures with 10-, 5- and 3-mm displacements in terms of load-to-failure. This suggests that different surgical approaches should be considered depending on the magnitude of displacement in such fractures. We have also shown that biomechanical classification of split fractures of HGT into Type I (single-fragment), Type II (singlefragment with medium size FT-RCT), Type III (multifragment) and Type IV (multi-fragment with medium size FT-RCT) fractures is reliable and can be used to guide the choice of surgical technique, thus confirming our primary hypothesis. To the best of our knowledge, this is the first study to describe the biomechanical differences between split fractures of the HGT using longterm clinical data.

Split fractures of the HGT involve lesions of the bone and rotator cuff matter. The present study revealed that Type IV fractures are most susceptible to failure following fixation with a shortened PHILOS plate. This is an important finding as it suggests that the number of fragments or inclusion of an RCT affects the final result of surgery. Furthermore, Type IV fractures were more likely to exhibit $10-\mathrm{mm}$ displacement. This highlights the necessity for clinicians to be aware of associated symptoms and to fix the RCT with a suture anchor at the same time as fixing the fracture. This information will enable improved preoperative planning and results in terms of shoulder function. Interestingly, there were no significant differences between groups among fractures with 3- or 5-mm displacement. This may be due to the use of a shortened PHILOS plate, which is a firm fixation. Significant differences between groups might become apparent if the fractures were fixed through fixation using doublerow sutures or the suture bridge technique. Future studies investigating the implications of different surgical techniques are warranted to evaluate the outcomes of different approaches.

There have been numerous studies focusing on indications for surgical treatment and fixation technique; however, only a few have focused on injuries involving both fragmentation and RCT [18-21]. An increasing number of studies have been published reporting injuries of the GT [22-24]; however, to the best of our knowledge, there have been none comparing single- and multi-fragment fractures. Some studies have examined associated injuries such as Bankart lesions, RCT and superior labral tear 
from anterior and posterior (SLAP) lesions in the context of these fractures [25]. Locking plate fixation provides superior fixation for split-type GT fractures compared with tension bands or double-row suture bridges. Therefore, we choose to use a shortened PHILOS plate for fixation in the present study. Previous studies have reported load-to-failure values of 842 or $1054 \mathrm{~N}$, considerably higher than the results of the present study [24]. This is likely due to the fact that we used formalin-fixed cadaver shoulder specimens.

Three main techniques of surgical fixation have been described for HGT fractures, with different techniques being more suitable for different types of fracture. Our study provides a system with which to classify fractures of the HGT and guide the choice of fixation technique. The specific recommendations that we propose are as follows: (i) Type I (single-fragment) fractures should be fixed using compression screws, which are inexpensive and efficient and have been shown to have favourable results through biomechanical studies. This is a useful approach for areas in which patients cannot afford high medical expenses and/or have insufficient health insurance and is also beneficial because the insertion angle can be adapted to increase biomechanical strength following fixation of osteoporotic fractures; a subject which warrants further study. However, compression screws may cause damage to fracture fragments $[7,16,24]$. (ii) Type II (single-fragment with medium size RCT) fractures should be fixed using screws combined with suture anchors under arthroscopic guidance. This method is widely used to treat PHFs as the tendon-bone interface fragment is fixed and satisfactory clinical results can be achieved [16, 18]. (iii) Type III (multi-fragment) fractures should be fixed using a suture bridge or small locking plate to provide stable fixation and early return to function. This surgical technique is simple and efficient [2628]. (iv) Type IV (multi-fragment with medium size RCT) fractures should be fixed using a small locking plate augmented with suture anchors via a mini-open deltoid-split approach [13, 16, 23, 24, 29].

The present study has some limitations which should be acknowledged. Firstly, we did not justify the use of this method of 3D reconstruction compared to other methods in patient with GT fracture. Due to a lack of human samples, we used 24 formalin-fixed specimens. Future studies should be carried out using fresh-frozen human cadaveric specimens. Secondly, the clinical models were not assessed by computed tomography scan or magnetic resonance imaging, which would have provided useful information. Thirdly, with the limited number of available cadaver specimens, we were unable to evaluate all known fixation techniques. With more specimens, further fixation techniques and the application of multiplanar ultimate loads could be evaluated.

\section{Conclusions}

The present study demonstrates that biomechanical classification of split fractures of the HGT is a reliable classification system. Although numerous surgical treatments for these fractures have been described, there is no gold standard in terms of treatment for this type of fracture. Therefore, our classification system will be a useful guide to enable surgeons to select an appropriate surgical technique. In the future work, we will validate this study with 3D Simulation (Finite element analysis).

\section{Abbreviations}

HGT: Humeral greater tuberosity; FT-RCT: Full-thickness rotator cuff tear; PHILOS: Proximal humeral internal locking system; PHFs: Proximal humeral fractures.

\section{Acknowledgements \\ Not applicable.}

\section{Author's contributions}

$\mathrm{LG}$ conducted the researches, wrote and revised the draft manuscript, and subsequent manuscript. GXG and ZQ designed the study, revised the draft manuscript. QB conducted the study, processed the figures, and participated in writing the draft manuscript. LJJ was contributing to the statistical analysis, the manuscript submission. BDS was contributing to the literature search. FSJ contributed the funds collection, and study design. All authors read and approved the final manuscript.

\section{Funding}

This study was supported by the Modern design and cultural research project of the research base on philosophy and Social Sciences of Sichuan in 2018 (Number: MD18E014).

\section{Availability of data and materials}

All data generated or analysed during this study are included in this published article and its supplementary information files.

\section{Declarations}

Ethics approval and consent to participate

All the procedures were approved by the Ethical Inspection Committee at Affiliated Traditional Chinese Medicine Hospital of Southwest Medical University (No. KYTL20180202) and performed according to the internationally accredited guidelines.

\section{Consent for publication}

Not applicable.

\section{Competing interests}

The authors declare that they have no competing interests.

\section{Author details}

${ }^{1}$ Department of Orthopedics, Affiliated Traditional Chinese Medicine Hospital of Southwest Medical University, Luzhou, Sichuan, China. ${ }^{2}$ Center for Orthopedic Diseases Research, Affiliated Traditional Chinese Medicine Hospital of Southwest Medical University, Luzhou, Sichuan, China. ${ }^{3}$ Expert Workstation in Luzhou, Luzhou, Sichuan, China. ${ }^{4}$ Clinical Base of Affiliated Traditional Chinese Medicine Hospital of Southwest Medical University, Guangdong Province Medical 3D Printing Application Transformation Engineering Technology Research Center, Luzhou, Sichuan, China. ${ }^{5}$ Department of Orthopedics, YiXing 
Traditional Chinese Medicine Hospital, Yixing, Jiangsu, China. ${ }^{6}$ Department of Breast Surgery, Luzhou Hospital of Traditional Chinese Medicine, Luzhou, Sichuan, China.

Received: 25 July 2021 Accepted: 10 November 2021 Published online: 24 November 2021

\section{References}

1. Baron JA, Karagas M, Barrett J, Kniffin W, Malenka D, Mayor M, Keller RB. Basic epidemiology of fractures of the upper and lower limb among Americans over 65 years of age. Epidemiology. 1996;7(6):612-8. https:// doi.org/10.1097/00001648-199611000-00008.

2. Geiger EV, Maier M, Kelm A, Wutzler S, Seebach C, Marzi I. Functional outcome and complications following PHILOS plate fixation in proximal humeral fractures. Acta Orthop Traumatol Turc. 2010;44(1):1-6. https:// doi.org/10.3944/aott.2010.2270

3. Bahrs C, Lingenfelter E, Fischer F, Walters EM, Schnabel M. Mechanism of injury and morphology of the greater tuberosity fracture. J Shoulder Elbow Surg. 2006;15(2):140-7. https://doi.org/10.1016/j.jse.2005.07.004.

4. Green A, Izzi J Jr. Isolated fractures of the greater tuberosity of the proximal humerus. J Shoulder Elbow Surg. 2003;12(6):641-9. https://doi.org/ 10.1016/s1058-2746(02)86811-2

5. Neer CS. 2nd Displaced proximal humeral fractures. I. Classification and evaluation. J Bone Joint Surg Am. 1970;52(6):1077-89.

6. Brunner A, Honigmann P, Treumann T, Babst R. The impact of stereo-visualisation of three-dimensional CT datasets on the inter- and intraobserver reliability of the AO/OTA and Neer classifications in the assessment of fractures of the proximal humerus. J Bone Joint Surg Br. 2009;91(6):76671. https://doi.org/10.1302/0301-620x.91b6.22109.

7. Mutch J, Laflamme GY, Hagemeister N, Cikes A, Rouleau DM. A new morphological classification for greater tuberosity fractures of the proximal humerus: validation and clinical implications. Bone Joint J. 2014:96b(5):646-51. https://doi.org/10.1302/0301-620x.96b5.32362.

8. Kim E, Shin HK, Kim CH. Characteristics of an isolated greater tuberosity fracture of the humerus. J Orthop Sci. 2005;10(5):441-4. https://doi.org/ 10.1007/s00776-005-0924-6.

9. Park TS, Choi IY, Kim YH, Park MR, Shon JH, Kim SI. A new suggestion for the treatment of minimally displaced fractures of the greater tuberosity of the proximal humerus. Bull Hosp Jt Dis. 1997;56(3):171-6.

10. Kim SH, Ha Kl. Arthroscopic treatment of symptomatic shoulders with minimally displaced greater tuberosity fracture. Arthroscopy. 2000;16(7):695-700. https://doi.org/10.1053/jars.2000.9237.

11. Gartsman GM, Taverna E. Arthroscopic treatment of rotator cuff tear and greater tuberosity fracture nonunion. Arthroscopy. 1996;12(2):242-4. https://doi.org/10.1016/s0749-8063(96)90021-3.

12. Gumina S, Carbone S, Postacchini F. Occult fractures of the greater tuberosity of the humerus. Int Orthop. 2009;33(1):171-4. https://doi.org/ 10.1007/s00264-007-0512-9.

13. Maman E, Dolkart O, Chechik O, Amar E, Rak O, Rath E, Mozes G. Arthroscopic findings of coexisting lesions with greater tuberosity fractures. Orthopedics. 2014;37(3):e272-277. https://doi.org/10.3928/0147744720140225-60.

14. Lin CL, Yeh WL, Su FC, Wang YC, Chiang CH, Hong CK, Su WR. Different suture anchor fixation techniques affect contact properties in humeral greater tuberosity fracture: a biomechanical study. BMC Musculoskelet Disord. 2020;20(1):26. https://doi.org/10.1186/s12891-019-2412-8.

15. Gaudelli C, Ménard J, Mutch J, Laflamme GY, Petit Y, Rouleau DM. Locking plate fixation provides superior fixation of humerus split type greater tuberosity fractures than tension bands and double row suture bridges.
Clin Biomech (Bristol, Avon). 2014;29(9):1003-8. https://doi.org/10.1016/j. clinbiomech.2014.08.013.

16. Cofield RH, Parvizi J, Hoffmeyer PJ, Lanzer WL, Ilstrup DM, Rowland CM. Surgical repair of chronic rotator cuff tears. A prospective long-term study. J Bone Joint Surg Am. 2001;83(1):71-7. https://doi.org/10.2106/ 00004623-200101000-00010.

17. Rouleau DM, Mutch J, Laflamme GY. Surgical treatment of displaced greater tuberosity fractures of the humerus. J Am Acad Orthop Surg. 2016:24(1):46-56. https://doi.org/10.5435/jaaos-d-14-00289.

18. Jabran A, Peach C, Zou Z, Ren L. Biomechanical comparison of screwbased zoning of PHILOS and Fx proximal humerus plates. BMC Musculoskelet Disord. 2018;19(1):253. https://doi.org/10.1186/s12891-018-2185-5.

19. Gruson KI, Ruchelsman DE, Tejwani NC. Isolated tuberosity fractures of the proximal humeral: current concepts. Injury. 2008;39(3):284-98. https://doi.org/10.1016/j.injury.2007.09.022.

20. George MS. Fractures of the greater tuberosity of the humerus. J Am Acad Orthop Surg. 2007;15(10):607-13. https://doi.org/10.5435/00124 635-200710000-00005.

21. Ji JH, Jeong JJ, Kim YY, Lee SW, Kim DY, Park SE. Clinical and radiologic outcomes of arthroscopic suture bridge repair for the greater tuberosity fractures of the proximal humerus. Arch Orthop Trauma Surg. 2017;137(1):9-17. https://doi.org/10.1007/s00402-016-2586-6.

22. Li R, Cai M, Tao K. Arthroscopic reduction and fixation for displaced greater tuberosity fractures using the modified suture-bridge technique. Int Orthop. 2017;41(6):1257-63. https://doi.org/10.1007/ s00264-017-3461-y.

23. Ji JH, Moon CY, Kim YY, Shafi M. Arthroscopic fixation for a malunited greater tuberosity fracture using the suture-bridge technique: technical report and literature review. Knee Surg Sports Traumatol Arthrosc. 2009;17(12):1473-6. https://doi.org/10.1007/s00167-009-0797-x.

24. Liao W, Zhang H, Li Z, Li J. Is arthroscopic technique superior to open reduction internal fixation in the treatment of isolated displaced greater tuberosity fractures? Clin Orthop Relat Res. 2016;474(5):1269-79. https:// doi.org/10.1007/s11999-015-4663-5.

25. Park SE, Jeong JJ, Panchal K, Lee JY, Min HK, Ji JH. Arthroscopic-assisted plate fixation for displaced large-sized comminuted greater tuberosity fractures of proximal humerus: a novel surgical technique. Knee Surg Sports Traumatol Arthrosc. 2016;24(12):3892-8. https://doi.org/10.1007/ s00167-015-3805-3

26. Braunstein V, Wiedemann E, Plitz W, Muensterer OJ, Mutschler W, Hinterwimmer S. Operative treatment of greater tuberosity fractures of the humerus-a biomechanical analysis. Clin Biomech (Bristol, Avon). 2007;22(6):652-7. https://doi.org/10.1016/j.clinbiomech.2007.03.002.

27. Hu C, Zhou K, Pan F, Zhai Q, Wen W, He X. Application of pre-contoured anatomic locking plate for treatment of humerus split type greater tuberosity fractures: a prospective review of 68 cases with an average follow-up of 2.5 years. Injury. 2018;49(6):1108-12. https://doi.org/10. 1016/j.injury.2018.04.013.

28. Zeng LQ, Chen YF, Jiang YW, Zeng LL, Miao XG, Liang WG. A new low profile anatomical locking plate for fixation of comminuted, displaced greater tuberosity fractures of the proximal humerus. J Shoulder Elbow Surg. 2020;30:1402. https://doi.org/10.1016/j.jse.2020.08.036.

29. Song HS, Williams GR Jr. Arthroscopic reduction and fixation with suturebridge technique for displaced or comminuted greater tuberosity fractures. Arthroscopy. 2008;24(8):956-60. https://doi.org/10.1016/j.arthro. 2008.01.009.

\section{Publisher's Note}

Springer Nature remains neutral with regard to jurisdictional claims in published maps and institutional affiliations. 